\title{
High Triglyceride-Glucose Index (TyG) is Associated With Adverse Cardiovascular Events in Patients With Type 2 Diabetes Mellitus (T2DM) and Chronic Total Occlusion (CTO) After Percutaneous Coronary Intervention (PCl)
}

Xiao Long Lin

Beijing An Zhen Hospital: Capital Medical University Affiliated Anzhen Hospital https://orcid.org/00000002-3331-7894

\section{Hao Xuan Sun}

Capital Medical University Affiliated Anzhen Hospital Jin Yang Zhao

Capital Medical University Affiliated Anzhen Hospital

Fan Qi Li

Capital Medical University Affiliated Anzhen Hospital

Dong Hui Zhao

Capital Medical University Affiliated Anzhen Hospital

Qin Ma

Capital Medical University Affiliated Anzhen Hospital Jing Hua Liu

Capital Medical University Affiliated Anzhen Hospital Qian Fan ( $\sim$ fanqian75@sina.com )

Capital Medical University

\section{Research}

Keywords: Triglyceride glucose index, Type 2 diabetes mellitus, chronic total occlusion, Percutaneous coronary intervention, Adverse cardiovascular outcomes

Posted Date: November 8th, 2021

DOI: https://doi.org/10.21203/rs.3.rs-1037669/v1

License: (c) (1) This work is licensed under a Creative Commons Attribution 4.0 International License. Read Full License 


\section{Abstract}

Background: The triglyceride-glucose index (TyG index) is a reliable surrogate marker of insulin resistance, which is associated with cardiovascular disease morbidity and prognosis. However, the predictive value of the TyG index for cardiovascular events in patients with type 2 diabetes mellitus (T2DM) and chronic total occlusion (CTO) after percutaneous coronary intervention (PCl) has not been specifically studied.

Method: The study retrospectively enrolled 687 patients with T2DM and CTO after PCl in the final analysis. Patients were divided into three groups according to the TyG index tertiles. The TyG index was calculated as $\ln$ [ fasting triglyceride $(\mathrm{mg} / \mathrm{dL}) \times$ fasting glucose $(\mathrm{mg} / \mathrm{dL}) / 2$ ]. The primary observational endpoint was the composite of overall death, non-fatal stroke, non-fatal myocardial infarction (MI), or unplanned revascularization.

Results: During a median follow-up of 22.0 months, 159 patients (23.14\%) experienced primary endpoint events. Multivariate Cox hazards regression analysis showed that the TyG index was significantly correlated with the primary endpoint [HR 2.827, 95\% Cl (1.877-4.529), P凶0.001]. Kaplan-Meier curves for the primary endpoint showed a significant difference between the lower and higher TyG index group was mainly driven by the increased incidence of unplanned revascularization (Log-rank Pख0.001).

Conclusion: The TyG index may be a remarkable predictor of adverse cardiovascular events, especially unplanned revascularization in patients with T2DM and CTO who are treated by PCl.

\section{Introduction}

Coronary artery disease (CAD) has one of the highest morbidity and mortality in the world. By 2030, according to data from the World Health Organization (WHO), the number of cardiovascular events in China will increase by about 73\% [1]. AS we know, Type 2 diabetes mellitus (T2DM) is a traditional risk factor of cardiovascular disease [2], chronic total occlusion (CTO) in type 2 diabetes mellitus (T2DM) patients is very common. Previous studies showed that this proportion is up to $34-40 \%[3,4]$. CTO with T2DM patients have more diffuse, anatomically complex coronary artery lesions and was associated with poor clinical outcomes. In clinical practice, we found that the poor prognosis of CTO with T2DM patients was different from those of common coronary heart disease, it is mainly reflected in ischemic heart disease, which leads to the occurrence of heart failure. Even if CTO vessels are successfully opened and coronary stents are implanted, Adverse cardiovascular events will still occur. At present, which indicators can predict the occurrence of cardiovascular events in T2DM patients with CTO after PCI is unknown. Therefore, it is essential to find a reliable method to identify the high-risk occurrence of MACE patients to provide intensive treatment.

The progression of insulin resistance not only leads to cardiovascular disease in patients with or without diabetes but also correlates with cardiovascular outcomes in patients with cardiovascular disease (CVD) $[5,6]$. TyG is a novel, convenient and inexpensive test to determine insulin resistance, which only needs to 
detect fasting glucose and triglyceride. It has been proved to be a significant correlation with HOMR which is used as a clinically useful surrogate measure of insulin resistance [7].

Many studies have shown that the TyG index is associated with cardiovascular disease morbidity and prognosis [8-11]; However, the prognostic value of the TyG index for cardiovascular events in patients with T2DM and CTO after PCI has not been specifically studied. In this sense, we studied the relationship between baseline TyG and cardiovascular outcomes in patients with T2DM and CTO who underwent PCI.

\section{Methods}

\section{Study population}

The present study is a single-center, retrospective observational cohort study. From January 2018 to December 2019, 687 consecutive patients with T2DM who were diagnosed with CTO and treated with PCI at Beijing An Zhen hospital were enrolled. The exclusion criteria were as follows: (1) Missing clinical data or follow-up data; (2) A prior coronary artery bypass grafting (CABG); (3) Left ventricular ejection fraction (LVEF) $<30 \%$; (4) Renal dysfunction with estimated glomerular filtration rate (eGFR) $<30 \mathrm{ml} /\left(\mathrm{min}^{\star 1.7 \rrbracket)}\right.$ or

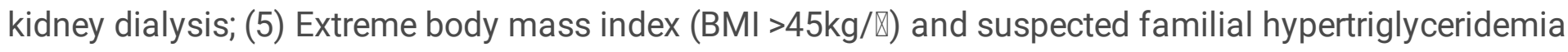
[plasma TGs $\geq 500 \mathrm{mg} / \mathrm{dl}$ (5.65mmol/L)]; (6) PCl failure or PCl-related complications; (7) Patients with a malignant tumor or other chronic diseases.

\section{Data collection and definitions}

Patients' data of demographic, clinical, and angiographic characteristics were collected from the medical information recording system of Beijing An Zhen Hospital.

The blood samples were collected after an overnight fast on admission and analyzed quickly after sampling. The routine hematology and biochemical parameters, including total cholesterol (TC), triglycerides (TG), low-density lipoprotein-C (LDL-C), high-density lipoprotein-C (HDL-C), FBG, glycosylated serum albumin, serum uric acid, serum creatinine, estimated glomerular filtration rate, and other biomarkers, were determined by standard laboratory methods in the central lab of Beijing Anzhen Hospital. The TyG index was calculated as the In (fasting TG level [mg/dL] × FBG level [mg/dL]/2) [12]. CTO was defined as a coronary arterial total occlusion with Thrombolysis In Myocardial Infarction (TIMI) flow grade 0 and the duration of occlusion $\geq 3$ months [13]. Overall death was defined as cardiovascular or non-cardiovascular causes. Unplanned revascularization was defined as the revascularization of the target vessel or non-target vessel.

\section{Follow-up details}

All patients were followed up every 6 months after baseline PCl. The follow-up period of this study lasted December 2020. The information about adverse events was obtained by trained professionals contacting patients or their families by telephone, who did not understand the baseline characteristics and were determined by carefully reviewing the corresponding medical records. 


\section{Endpoints}

The primary observational endpoint was the composite of overall death, non-fatal stroke, non-fatal myocardial infarction (MI), or unplanned revascularization.

\section{Statistical analysis}

Continuous variables were presented as the mean \pm standard deviation is consistent with a normal distribution, otherwise as to the median and interquartile range (IQR). Categorical variables were expressed as numbers and percentages. The ANOVA or Kruskal-Wallis tests were used to analyze differences in continuous variables. The Pearson chi-square test (Pearson $X^{2}$ test) or Fisher's exact test was used to analyze categorical variables. The correlation between the TyG index and traditional cardiovascular risk factors was evaluated by Pearson correlation analysis. Receiver-operating characteristic (ROC) curve analysis was used to predict the value of the TyG index for the primary endpoint. The Kaplan-Meier survival analyses were performed to evaluate the incidence rate of primary endpoint between groups according to the TyG index tertiles, and otherness between groups was evaluated by a log-rank test. Variables with a univariate $P$ value $<0.05$ were selected for multivariate analysis. Multivariate cox proportional hazards regression analysis to estimate whether TyG is a predictor of adverse cardiovascular events and to identify other factors related to outcome in patients with T2DM and CTO who were treated by PCl. The predictive value of the TyG index for each component of the primary endpoint was also evaluated by univariate and multivariate Cox regression analysis. Since the TyG index was calculated according to FBG and TGs, these two variables were not taken into multivariate analysis. All statistical analysis results were two-tailed and a p-value $<0.05$ was regarded as statistically significant. Data were analyzed by IBM SPSS statistics 24 .

\section{Result}

As shown in Figure.1, Six hundred and eighty-seven patients (mean age: $59.1 \pm 9.8$ years; $82.82 \%$ men) were enrolled in the final analysis. Throughout a median of 22.0 months follow-up, 20 patients (of the total population) were lost to follow-up. $159(23.14 \%)$ patients intimate primary terminus events, as well as $10(1.46 \%)$ overall cause death, $30(4.41 \%)$ non-fatal $\mathrm{Ml}$, and $109(15.87 \%)$ unplanned revascularization.

\section{Baseline characteristics of patients}

According to the TyG index tertiles, baseline characteristics of groups were presented in Table 1. As TyG index elevated, body mass index, TGs, TC, LDL-C, uric acid, FBG, Glycosylated serum protein (GA), and Insulin used significantly increased, while HDL-C levels and the number of LAD lesions were decreased. The ROC curves of the triglyceride-glucose index as a marker to predict the primary endpoint in CTO patients after PCl were presented in Figure.2. The AUC of the TyG index for forecasting the occurrence of the primary endpoint in CTO patients was 0.623 (95\% $\mathrm{Cl} 0.575-0.671 ; \mathrm{P} \otimes 0.001)$. The baseline characteristics of the groups stratified by the primary endpoint are shown in Table 2. Compared 
with the without events group, the Primary endpoint group had a higher proportion of males, smoking, drinking, Previous MI, Past PCl, Previous Stroke, and had higher Uric acid, FPG, TyG index, TC, TG, LDL, lower blood pressure. In these variables, Only FBG, TGs, TyG index, Uric acid were independently associated with the primary endpoint group. The other variables were no statistical significance.

\section{The TyG index had a higher correlation with traditional cardiovascular risk factors}

The result of the Pearson correlation analysis of the correlation between the TyG index and traditional cardiovascular risk factors is shown in Table 3. TyG index had a higher correlation with BMI, Glycosylated serum protein (GA), TGs, FBG, LDL-C, TC, uric acid, and negatively associated with Age, HDL-C, and LVEF (all $P<0.05)$.

\section{The TyG index was significantly correlated with the primary endpoint}

The univariate and multivariate Cox proportional hazard analysis for the primary endpoint is shown in Table 4. The univariate analysis showed that TyG index, Previous MI, TGs, FPG, oral P2Y12 inhibitors agent, uric acid, insulin injection were risk factors for adverse cardiovascular events in patients with T2DM and CTO after PCI (all Pख0.05). To determine whether TyG is an independent risk factor for adverse cardiovascular events and to identify other factors related to outcome, We performed a multivariate analysis. After adjusting variables with a univariate $P$ value $₫ 0.05$, Multivariate analysis revealed that both the TyG index and oral P2Y12 inhibitors agent were significantly correlated with the primary endpoint (P区 0.05). The incidence of the primary endpoint and each component according to the TyG index tertiles is shown in Table 5. Compared with the Q1 group, Both the primary endpoint and unplanned repeat revascularization increased significantly in Q2, Q3 group (chi-square $\mathrm{P}<0.001$ ), while the overall death, non-fatal stroke, non-fatal MI were without Statistical significance between the three groups. To further verify the reliability and stability of the results, we evaluated the predictive value of the TyG index as a nominal and continuous variable respectively for each component of the primary endpoint by univariate and multivariate Cox proportional hazard analysis. In univariate analysis, TyG index as a nominal and taking Q1 as the reference冈the result showed that Q2, Q3 group were associated with the primary endpoint ( Q2: HR 1.675, 95\% Cl 1.081-2.596, P= 0.021; Q3: HR 2.827, 95\% Cl 1.877-4.529, Pख0.001; ) and Q3 group was associated with unplanned revascularization ( Q2: HR $1.53595 \% \mathrm{Cl} 0.882-2.672, \mathrm{P}=0.129$; Q3: HR 3.115, 95\% $\mathrm{Cl} 1.888-5.139, \mathrm{P} \otimes 0.001$; ). However, the higher TyG index is unable to predict the overall death, non-fatal stroke, and non-fatal MI. After adjusting for age, gender, and other confounding factors, Multivariate analysis showed that the incidence of the primary endpoint ( Q2: HR $1.77895 \% \mathrm{Cl}$ 1.124-2.813, $\mathrm{P}=0.014$; Q3: HR 3.199, 95\% $\mathrm{Cl} 2.000-5.118, \mathrm{P} \otimes 0.001$; ) and unplanned repeat revascularization ( Q2: HR $1.61095 \% \mathrm{Cl} 0.900-2.883, \mathrm{P}=0.109$; Q3: HR 3.743, 95\% Cl 2.113-6.63, P区 0.001 ; ) were increased as the TyG increase, while the Q2 and Q3 group fail to predict the overall death, non-fatal stroke and non-fatal MI. When the TyG index was a continuous variable, either the univariate or multivariate analysis, the result was similar to the TyG index as a nominal variable (Table 5).

The higher TyG index group is significantly associated with the primary endpoint and unplanned revascularization. 
Kaplan-Meier curves for the incidence of primary endpoint and each component of it according to the TyG index tertiles were shown in Figure.3. Kaplan-Meier curves showed that the Q3 group had a higher risk for the primary endpoint (Fig.3a, Log-rank $\mathrm{P}<0.001$ ) and unplanned revascularization (Fig.3d, Logrank $P<0.001$ ). However, there is no statistical significance for overall-cause death (Fig. 3b, Log-rank $P$ $=0.88$ ), non-fatal myocardial infarction (Fig.3c, Log-rank $P=0.27$ ), and non-fatal stroke (Fig. 3e, Log-rank $P=0.73$ ) between the three groups.

\section{Discussion}

The presence of CTO lesions is the strongest independent predictor of incomplete revascularization among patients treated with $\mathrm{PCl}$ [14]. Patients with T2DM and incomplete revascularization have an increased risk of long-term cardiovascular events including death, $\mathrm{Ml}$, stroke, and repeat revascularization [15]. Although CTO vessels are successfully opened, Diabetic patients remain at increased risk for adverse cardiovascular events following $\mathrm{PCl}$, driven by higher rates of target lesion revascularization (TLR) [16]. Our study sought a novel method to identify the high-risk occurrence of MACE in T2DM patients with $\mathrm{CTO}$ undergoing $\mathrm{PCl}$.

Our main findings include: (1) TyG is significantly associated with the primary endpoint, which was composed of non-fatal myocardial infarction, non-fatal stroke, overall death, and unplanned revascularization in T2DM patients with CTO after PCl. (2) TyG is correlated with multiple cardiovascular disease risk factors. (3) The predictive effect of the TyG index on the compound primary endpoint was mainly reflected in the unplanned revascularization.

Diabetes is a major risk factor for cardiovascular disease, which is common in patients with CTO. But Only $35 \%$ of the additional cardiovascular risk caused by T2DM is mediated by traditional cardiovascular risk factors. The most enormous mediating effect is insulin resistance [17]. Insulin resistance is a major component of metabolic syndrome, type 2 diabetes, and cardiovascular disease (CVD) [18]. The gold standard for measuring insulin resistance is the hyperinsulinemic-euglycemic glucose clamp technique which is mainly used in clinical research to limit its clinical application. Homoeostasis model assessment (HOMA) and QUICKI were widely used as clinically useful surrogate insulin resistance measures [18]. However, it costs a lot of money, takes a long time to test, and insulin level is not a routine clinical test, which can't be used in a wide range of clinical applications. Therefore, convenient and inexpensive tests are needed to determine insulin resistance. TyG is a novel surrogate marker of insulin resistance, which only detects fasting glucose and triglyceride. It has been proved to be a significant correlation with HOMR [21], but it is faster, more convenient, and less expensive than HOMR.

The mechanism of cardiovascular disease caused by insulin resistance can be summarized into three aspects. (i) Insulin signaling is key to the activation of nitric oxide, an effective vasodilator dose, and antiatherosclerosis [22, 23], Insulin resistance leads to impaired insulin signaling, which leads to high blood pressure and atherosclerosis. (ii) Insulin resistance causes compensatory hyperinsulinemia, Hyperinsulinemia can stimulate the synthesis and secretion of very-low-density lipoprotein (LDL) [24], 
stimulate vascular smooth muscle cell proliferation [25], promote the release of inflammatory factors [26], and enhance LDL cholesterol transport to arterial smooth muscle cells [27]. (iii) Insulin resistance is associated with Metabolic syndrome, Each component of the Metabolic syndrome is an independent risk factor for ASCVD [28].

Many studies have shown that the TyG index is associated with cardiovascular disease morbidity and prognosis. Da, S.A. et al. evaluated the correlation between the TyG index and coronary artery disease, as well as cardiovascular risk factors, showed that the TyG index was positively correlated with a higher prevalence of symptomatic CAD, which could be used as a marker for atherosclerosis [8]. The study from Luo et al. enrolled 1092 STEMI patients after PCI to assess the role of the TyG index as a predictor of prognosis in STEMI patients after PCl. According to the TyG index, The patients were divided into 4 quartiles. The results suggested that the TyG index might be an effective predictor of clinical outcomes in STEMI patients undergoing $\mathrm{PCl}(\mathrm{HR} 1.52995 \% \mathrm{Cl} 1.001-2.061 ; \mathrm{P}=0.003)$ [9]. Another observational study from Mao et al. showed that TyG index was not only an independent predictor of coronary artery severity and cardiovascular disease prognosis in patients with acute non-ST-segment elevation myocardial infarction but also had strongly independently associated with SYNTAX score (OR 6.055, 95\% Cl 2.915$12.579, P<0.001)$ [10]. A recent study from $\mathrm{Ma}, \mathrm{X}$., et al. included 776 patients with T2DM and ACS who underwent $\mathrm{PCl}$ to research the prognostic usefulness of the TyG index in patients with T2DM and ACS after $\mathrm{PCl}$. The result suggested that TyG may be a valuable predictor of adverse cardiovascular outcomes after PCl in patients with T2DM and ACS [11]. Previous research had also shown that the TyG index was a significant correlation with subclinical CAD in healthy individuals [29], the progression of coronary artery calcification [30-32], the macro-and microvascular damage [33], the arterial stiffness [34], hypertension [35], the number and the severity of artery stenoses [36]. However, the effects of the TyG index on cardiovascular prognosis in T2DM patients with CTO after PCl are still unclear.

Our study showed that the major MACE rates among T2DM patients with CTO after PCl were caused by unplanned revascularization, which is consistent with the Sohrabi et al. study[37], and the higher TyG is a significant correlation with unplanned revascularization. We found that the TyG index was a positive correlation with BMI, GA, LDL-C, TC, uric acid, and was negatively associated with HDL-C. Furthermore, these variables were significantly higher in patients in the Q3 group than Q1, Q2 group. These factors are traditional risk factors for cardiovascular disease, which partly explains why TyG can predict cardiovascular disease outcome events.

\section{Limitation}

This study had several limitations. First, this study was a single-center, retrospective study, which may have a selection bias or potential confounding factors. Meanwhile, the small sample size of this study may weaken the conviction of the results. Second, the experimental parameters were only measured at admission, and the dynamic changes of TyG were not monitored during the follow-up period, which may result in measurement bias. Third, Some patients have used lipid-lowering and hypoglycemic drugs before admission, which may affect the results of triglyceride and fasting blood glucose. Fourth, We did 
not routinely calculate the SYNTAX score, so we did not evaluate the correlation between the TYG index and syntax score.

\section{Conclusion}

The TyG index, which is a novel, simple and reliable surrogate marker of insulin resistance, maybe a remarkable predictor of adverse cardiovascular events, especially revascularization in type 2 diabetic patients after CTO PCI. Further prospective, large, multicenter studies are needed to confirm our findings.

\section{Abbreviations}

Type 2 Diabetes Mellitus, T2DM; Chronic Total Occlusion, CTO; BMI, body mass index; SBP, systolic blood pressure; DBP, diastolic blood pressure; MI, myocardial infarction; $\mathrm{PCl}$, percutaneous coronary intervention; CKD, chronic kidney disease; LVEF, left ventricular ejection fraction; TC, total cholesterol; LDL-C, lowdensity lipoprotein cholesterol; HDL-C, high-density lipoprotein cholesterol; TGs, triglycerides; FBG, fasting blood glucose; GA, glycated albumin; TyG, triglyceride glucose; eGFR, estimated glomerular filtration rate; $A C E l$, angiotensin-converting enzyme inhibitor; $A R B$, angiotensin receptor blocker; $C C B$, Calcium Channel Blockers; LM, left main artery; LAD, left anterior descending artery; LCX, left circumflex artery; RCA, right coronary artery $\mathbb{Q L V E F}$, left ventricular ejection fraction; $\mathrm{HR}$, hazard ratio; $\mathrm{Cl}$, confidence interval.

\section{Declarations}

\section{Ethics approval and consent to participate]}

The present study was performed by the Helsinki Declaration of Human Rights and approved by the Clinical Research Ethics Committee of Beijing An Zhen Hospital, Capital Medical University. Given the retrospective nature of this study, and written informed consent was obtained from all patients.

\section{Consent for publication $\square$}

Not applicable.

\section{Availability of data and materials $\square$}

The datasets used and/or analyzed during the present study are available from the corresponding author on reasonable request.

\section{Competing interests:}

The authors declare no potential conflict of interest.

\section{Funding}


This work was supported by the National Natural Sciences Foundation of China (NSFC) [grants \#81971302 and \#81771494 (both to Qian Fan)].

\section{Authors' contributions:}

XLL takes responsibility for all aspects of the reliability and freedom from bias of the data presented and their discussed interpretation. QF, XLL takes responsibility for research design. HXS, JYZ, FQ, take responsibility for data collection. $D H Z \triangle Q M \otimes J H L$ takes responsibility for the data review. All authors read and approved the final manuscript.

\section{Acknowledgments:}

We are very grateful to the medical staff in Ward 28 of the Department of Cardiology, Beijing Anzhen Hospital, Capital Medical University for their support to this study.

\section{References}

1. WHO, 2016. Cardiovascular Diseases. Available at:

http://www.wpro.who.int/china/mediacentre/factsheets/cvd/en/. Accessed 17 January 2016.

2. Norhammar, A., et al., Diabetes mellitus: the major risk factor in unstable coronary artery disease even after consideration of the extent of coronary artery disease and benefits of revascularization. $J$ Am Coll Cardiol, 2004. 43(4): p. 585-91.

3. Salisbury, A.C., et al., Outcomes of Chronic Total Occlusion Percutaneous Coronary Intervention in Patients With Diabetes: Insights From the OPEN CTO Registry. JACC Cardiovasc Interv, 2017. 10(21): p. 2174-2181.

4. Choi, K.H., et al., Long-term clinical outcomes of patients with coronary chronic total occlusion treated with percutaneous coronary intervention versus medical therapy according to presence of diabetes mellitus. Eurolntervention, 2017. 13(8): p. 970-977.

5. Nishimura, M., et al., Association of insulin resistance with de novo coronary stenosis after percutaneous coronary artery intervention in hemodialysis patients. Nephron Clin Pract, 2008. 109(1): p. c9-17.

6. Uetani, T., et al., Impact of insulin resistance on post-procedural myocardial injury and clinical outcomes in patients who underwent elective coronary interventions with drug-eluting stents. JACC Cardiovasc Interv, 2012. 5(11): p. 1159-67.

7. Guerrero-Romero, F., et al., The product of triglycerides and glucose, a simple measure of insulin sensitivity. Comparison with the euglycemic-hyperinsulinemic clamp. J Clin Endocrinol Metab, 2010. 95(7): p. 3347-51.

8. Da, S.A., et al., Triglyceride-glucose index is associated with symptomatic coronary artery disease in patients in secondary care. Cardiovasc Diabetol, 2019. 18(1): p. 89. 
9. Luo, E., et al., High triglyceride-glucose index is associated with poor prognosis in patients with acute ST-elevation myocardial infarction after percutaneous coronary intervention. Cardiovasc Diabetol, 2019. 18(1): p. 150.

10. Mao, Q., et al., The Triglyceride-Glucose Index Predicts Coronary Artery Disease Severity and Cardiovascular Outcomes in Patients with Non-ST-Segment Elevation Acute Coronary Syndrome. Dis Markers, 2019. 2019: p. 6891537.

11. Ma, X., et al., Triglyceride glucose index for predicting cardiovascular outcomes after percutaneous coronary intervention in patients with type 2 diabetes mellitus and acute coronary syndrome. Cardiovasc Diabetol, 2020. 19(1): p. 31.

12. Guerrero-Romero, F., et al., The product of triglycerides and glucose, a simple measure of insulin sensitivity. Comparison with the euglycemic-hyperinsulinemic clamp. J Clin Endocrinol Metab, 2010. 95(7): p. 3347-51.

13. Galassi, A.R., et al., Percutaneous recanalisation of chronic total occlusions: 2019 consensus document from the EuroCTO Club. Eurolntervention, 2019. 15(2): p. 198-208.

14. Farooq, V., et al., The negative impact of incomplete angiographic revascularization on clinical outcomes and its association with total occlusions: the SYNTAX (Synergy Between Percutaneous Coronary Intervention with Taxus and Cardiac Surgery) trial. J Am Coll Cardiol, 2013. 61(3): p. 28294.

15. Schwartz, L., et al., Impact of completeness of revascularization on long-term cardiovascular outcomes in patients with type 2 diabetes mellitus: results from the Bypass Angioplasty Revascularization Investigation 2 Diabetes (BARI 2D). Circ Cardiovasc Interv, 2012. 5(2): p. 166-73.

16. Koskinas, K.C., et al., Impact of Diabetic Status on Outcomes After Revascularization With DrugEluting Stents in Relation to Coronary Artery Disease Complexity: Patient-Level Pooled Analysis of 6081 Patients. Circ Cardiovasc Interv, 2016. 9(2): p. e003255.

17. Sharif, S., et al., Mediation analysis of the relationship between type 2 diabetes and cardiovascular events and all-cause mortality: Findings from the SMART cohort. Diabetes Obes Metab, 2019. 21(8): p. 1935-1943.

18. Bloomgarden, Z.T., Insulin resistance concepts. Diabetes Care, 2007. 30(5): p. 1320-6.

19. Matthews, D.R., et al., Homeostasis model assessment: insulin resistance and beta-cell function from fasting plasma glucose and insulin concentrations in man. Diabetologia, 1985. 28(7): p. 412-9.

20. Katz, A., et al., Quantitative insulin sensitivity check index: a simple, accurate method for assessing insulin sensitivity in humans. J Clin Endocrinol Metab, 2000. 85(7): p. 2402-10.

21. Guerrero-Romero, F., et al., The product of triglycerides and glucose, a simple measure of insulin sensitivity. Comparison with the euglycemic-hyperinsulinemic clamp. J Clin Endocrinol Metab, 2010. 95(7): p. 3347-51.

22. Defronzo, R.A., Banting Lecture. From the triumvirate to the ominous octet: a new paradigm for the treatment of type 2 diabetes mellitus. Diabetes, 2009. 58(4): p. 773-95. 
23. DeFronzo, R.A., Insulin resistance, lipotoxicity, type 2 diabetes and atherosclerosis: the missing links. The Claude Bernard Lecture 2009. Diabetologia, 2010. 53(7): p. 1270-87.

24. Lucero, D., et al., Overproduction of altered VLDL in an insulin-resistance rat model: Influence of SREBP-1c and PPAR-a. Clin Investig Arterioscler, 2015. 27(4): p. 167-74.

25. Golovchenko, I., et al., Hyperinsulinemia enhances transcriptional activity of nuclear factor-kappaB induced by angiotensin II, hyperglycemia, and advanced glycosylation end products in vascular smooth muscle cells. Circ Res, 2000. 87(9): p. 746-52.

26. Choubey, A., et al., Low-dose naltrexone rescues inflammation and insulin resistance associated with hyperinsulinemia. J Biol Chem, 2020. 295(48): p. 16359-16369.

27. Porter, K.E. and K. Riches, The vascular smooth muscle cell: a therapeutic target in Type 2 diabetes? Clin Sci (Lond), 2013. 125(4): p. 167-82.

28. Ormazabal, V., et al., Association between insulin resistance and the development of cardiovascular disease. Cardiovasc Diabetol, 2018. 17(1): p. 122.

29. Park, G.M., et al., Triglyceride glucose index is a useful marker for predicting subclinical coronary artery disease in the absence of traditional risk factors. Lipids Health Dis, 2020. 19(1): p. 7.

30. Kim, M.K., et al., Relationship between the triglyceride glucose index and coronary artery calcification in Korean adults. Cardiovasc Diabetol, 2017. 16(1): p. 108.

31. Won, K.B., et al., Triglyceride glucose index is an independent predictor for the progression of coronary artery calcification in the absence of heavy coronary artery calcification at baseline. Cardiovasc Diabetol, 2020. 19(1): p. 34.

32. Park, K., et al., Elevated TyG Index Predicts Progression of Coronary Artery Calcification. Diabetes Care, 2019. 42(8): p. 1569-1573.

33. Zhao, S., et al., Association between macro- and microvascular damage and the triglyceride glucose index in community-dwelling elderly individuals: the Northern Shanghai Study. Cardiovasc Diabetol, 2019. 18(1): p. 95.

34. Lee, S.B., et al., Association between triglyceride glucose index and arterial stiffness in Korean adults. Cardiovasc Diabetol, 2018. 17(1): p. 41.

35. Zhu, B., et al., A high triglyceride glucose index is more closely associated with hypertension than lipid or glycemic parameters in elderly individuals: a cross-sectional survey from the Reaction Study. Cardiovasc Diabetol, 2020. 19(1): p. 112.

36. Thai, P.V., et al., Triglyceride glucose index for the detection of asymptomatic coronary artery stenosis in patients with type 2 diabetes. Cardiovasc Diabetol, 2020. 19(1): p. 137.

37. Sohrabi, B., et al., Outcome of diabetic and non-diabetic patients undergoing successful percutaneous coronary intervention of chronic total occlusion. J Cardiovasc Thorac Res, 2011. 3(2): p. 45-8.

\section{Tables}


Table 1 Baseline clinical characteristics of patients according to the TyG index tertiles

Note:Values are presented as the mean \pm SD, median (IQR) or number (\%).

The groups were stratified by tthe TyG index tertiles

Abbreviation:BMI, body mass index; SBP, systolic blood pressure; DBP, diastolic blood pressure; MI, myocardial infarction; $\mathrm{PCl}$, percutaneous coronary intervention; $\mathrm{CKD}$, chronic kidney disease; LVEF, left ventricular ejection fraction; TC, total cholesterol; LDL-C, low-density lipoprotein cholesterol; HDL-C, highdensity lipoprotein cholesterol; TGs, triglycerides; FBG, fasting blood glucose; GA, glycated albumin; TyG, triglyceride glucose; eGFR, estimated glomerular filtration rate; $\mathrm{ACEl}$, angiotensin converting enzyme inhibitor; ARB, angiotensin receptor blocker; CCB, Calcium Channel Blockers; LM, left main artery; LAD, left anterior descending artery; LCX, left circumflex artery; RCA, right coronary artery.

Table 2 Baseline clinical characteristics of patients stratified by the primary ednpoint

Table 2 (continues)

Note:Values are presented as the mean \pm SD, median (IQR) or number (\%).

Abbreviation:BMI, body mass index; SBP, systolic blood pressure; DBP, diastolic blood pressure; MI, myocardial infarction; $\mathrm{PCl}$, percutaneous coronary intervention; $\mathrm{CKD}$, chronic kidney disease; LVEF, left ventricular ejection fraction; TC, total cholesterol; LDL-C, low-density lipoprotein cholesterol; HDL-C, highdensity lipoprotein cholesterol; TGs, triglycerides; FBG, fasting blood glucose; GA, glycated albumin; TyG, triglyceride glucose; eGFR, estimated glomerular filtration rate; ACEI, angiotensin converting enzyme inhibitor; ARB, angiotensin receptor blocker; CCB, Calcium Channel Blockers; LM, left main artery; LAD, left anterior descending artery; LCX, left circumflex artery; RCA, right coronary artery.

Table 3 Correlations between the TyG index and traditional cardiovascular risk factors

Abbreviation:BMI, body mass index; FBG, fasting blood glucose; GA, glycated albumin; TC, total cholesterol; LDL-C, low-density lipoprotein cholesterol; HDL-C, high-density lipoprotein cholesterol; eGFR, estimated glomerular filtration rate; LVEF, left ventricular ejection fraction; TGs, triglycerides;

Table 4 Relationship between the incidence of the primary endpoint and the TyG index

Abbreviation:BMI, body mass index; SBP, systolic blood pressure; DBP, diastolic blood pressure; MI, myocardial infarction; $\mathrm{PCl}$, percutaneous coronary intervention; $\mathrm{CKD}$,chronic kidney disease; LVEF, left ventricular ejection fraction; TC, total cholesterol; LDL-C, low-density lipoprotein cholesterol; HDL-C, highdensity lipoprotein cholesterol; TGs, triglycerides; FBG, fasting blood glucose; GA, glycated albumin; TyG, 


\begin{tabular}{|c|c|c|c|c|}
\hline & Q1 ( n=229) & Q2 ( $n=229)$ & Q3 ( n=229) & $P$ \\
\hline \multicolumn{5}{|l|}{ Demographics } \\
\hline Age,years & $60.25 \pm 9.59$ & $58.70 \pm 10.07$ & $58.41 \pm 9.72$ & 0.097 \\
\hline Male Sex,nヌ\%区 & $191(83.4)$ & $194(84.7)$ & $184(80.3)$ & 0.446 \\
\hline $\mathrm{BMI}, \mathrm{kg} / \mathrm{m}^{2}$ & $25.37 \pm 3.15$ & $26.82 \pm 3.13$ & $27.18 \pm 3.15$ & $<0.001$ \\
\hline \multicolumn{5}{|l|}{$\begin{array}{l}\text { Medical } \\
\text { measurements }\end{array}$} \\
\hline $\mathrm{SBP}, \mathrm{mmHg}$ & $128 \pm 16$ & $128 \pm 16$ & $128 \pm 15$ & 0.930 \\
\hline $\mathrm{DBP}, \mathrm{mmHg}$ & $76 \pm 11$ & $76 \pm 10$ & $77 \pm 10$ & 0.506 \\
\hline \multicolumn{5}{|l|}{ Risk factors } \\
\hline Smoking,n(\%) & $0.52(0.50)$ & $0.53(0.50)$ & $0.54(0.50)$ & 0.895 \\
\hline drinkers,n(\%) & $67(29.3)$ & $62(27.1)$ & $58(25.3)$ & 0.639 \\
\hline Hypertension,n(\%) & $154(67.2)$ & $152(66.4)$ & $164(71.6)$ & 0.434 \\
\hline Dyslipidaemia,n(\%) & $165(72.1)$ & $160(69.9)$ & $154(67.2)$ & 0.534 \\
\hline Previous MI,n(\%) & $64(27.9)$ & $56(24.5)$ & $73(31.9)$ & 0.209 \\
\hline Past PCl,n(\%) & $77(33.6)$ & $74(32.3)$ & $87(38.0)$ & 0.409 \\
\hline Previous Stroke,n(\%) & $24(10.5)$ & $17(7.4)$ & $24(10.5)$ & 0.435 \\
\hline CKD,n(\%) & $3(1.3)$ & $6(2.6)$ & $8(3.5)$ & 0.318 \\
\hline LVEF(\%) & $61 \pm 8$ & $59 \pm 9$ & $59 \pm 8$ & 0.010 \\
\hline \multicolumn{5}{|l|}{ Laboratory findings } \\
\hline $\mathrm{TC}(\mathrm{mg} / \mathrm{dL})$ & $138.79 \pm 35.81$ & $147.82 \pm 40.68$ & $158.72 \pm 39.77$ & $<0.001$ \\
\hline LDL-C (mg/dL) & $81.14 \pm 32.68$ & $88.13 \pm 36.06$ & $89.00 \pm 32.44$ & 0.024 \\
\hline $\mathrm{HDL}-\mathrm{C}(\mathrm{mg} / \mathrm{dL})$ & $38.22 \pm 13.81$ & $38.21 \pm 14.04$ & $36.62 \pm 11.94$ & 0.332 \\
\hline Triglycerides (mg/dL) & $88.0(67.0,104.0)$ & $143.0(121.0,163.0)$ & $212.0(160.0,282.0)$ & $<0.001$ \\
\hline $\mathrm{FPG}(\mathrm{mg} / \mathrm{dL})$ & $98.0(91.0,111.0)$ & $112.0(98.0,134.0)$ & $155.0(123.0,201.0)$ & $<0.001$ \\
\hline $\mathrm{GA}, \%$ & $15.0(13.0,17.0)$ & $16.0(14.0,18.0)$ & $18.0(15.0,22.0)$ & $<0.001$ \\
\hline TyG index & $8.0(8.0,9.0)$ & $9.0(9.0,9.0)$ & 10.0(9.0,10.0区 & $<0.001$ \\
\hline eGFR, $\mathrm{mL} / \mathrm{min}$ & $94.92 \pm 16.63$ & $93.52 \pm 15.62$ & $95.09 \pm 15.93$ & 0.516 \\
\hline Uric acid,umol/L & $346.21 \pm 82.30$ & $372.78 \pm 96.68$ & $371.91 \pm 91.90$ & 0.002 \\
\hline
\end{tabular}




\begin{tabular}{|c|c|c|c|c|}
\hline $\begin{array}{l}\text { Medications at } \\
\text { discharge }\end{array}$ & & & & \\
\hline Aspirin, n (\%) & $228(99.6)$ & $225(98.3)$ & $229(100.0)$ & 0.073 \\
\hline P2Y12 inhibitors, $\mathrm{n}(\%)$ & $145(63.3)$ & $153(66.8)$ & $162(70.7)$ & 0.240 \\
\hline Statin,n(\%) & $177(77.3)$ & $179(78.2)$ & $191(83.4)$ & 0.214 \\
\hline ACEI/ARBs, $n(\%)$ & $75(32.8)$ & $71(31.0)$ & $82(35.8)$ & 0.543 \\
\hline$\beta$-blockers, n (\%) & $113(49.3)$ & $116(50.7)$ & $137(59.8)$ & 0.050 \\
\hline Insulin, n (\%) & $13(5.7)$ & $19(8.3)$ & $35(15.3)$ & 0.002 \\
\hline $\mathrm{CCB}, \mathrm{n}(\%)$ & $32(14.0)$ & $23(10.0)$ & $31(13.5)$ & 0.379 \\
\hline Angiographic findings & & & & \\
\hline $\begin{array}{l}\text { One-vessel } \\
\text { disease,n(\%) }\end{array}$ & $51(22.3)$ & $42(18.3)$ & $41(17.9)$ & 0.430 \\
\hline $\begin{array}{l}\text { Two-vessel } \\
\text { disease,n(\%) }\end{array}$ & $64(27.9)$ & $54(23.6)$ & $62(27.1)$ & 0.531 \\
\hline $\begin{array}{l}\text { three-vessel } \\
\text { disease,n(\%) }\end{array}$ & $114(49.8)$ & $130(56.8)$ & $125(54.6)$ & 0.308 \\
\hline Target vessel territory & & & & \\
\hline LAD, n (\%) & $104(45.4)$ & $87(38.0)$ & 77 (33.6) & 0.033 \\
\hline LCX, n (\%) & $41(17.9)$ & $51(22.3)$ & $47(20.5)$ & 0.504 \\
\hline RCA, n (\%) & $120(52.4)$ & 133(58.1) & $134(58.5)$ & 0.339 \\
\hline
\end{tabular}

triglyceride glucose; eGFR, estimated glomerular filtration rate; $\mathrm{ACEl}$, angiotensin converting enzyme inhibitor; ARB, angiotensin receptor blocker; CCB, Calcium Channel Blockers; LM, left main artery; LAD, left anterior descending artery; LCX, left circumflex artery; RCA, right coronary artery.

Table 5 Incidence of primary endpoint and each compenents according to the TyG index tertiles Note: The groups were stratified by the TyG index tertiles Abbreviation: TyG triglyceride glucose, MI myocardial infarction Table 6 Univariable and Multivariable Cox regression analysis of Primary endpoint 


\begin{tabular}{|c|c|c|c|}
\hline & Without events $(n=528)$ & Primary endpoint $(n=159)$ & $P$ value \\
\hline \multicolumn{4}{|l|}{ Demographics } \\
\hline Age,years & $59.02 \pm 9.49$ & $59.44 \pm 10.84$ & 0.640 \\
\hline Male Sex,n $\rrbracket \% \rrbracket$ & $434(82.2)$ & $135(84.9)$ & 0.500 \\
\hline $\mathrm{BMI}, \mathrm{kg} / \mathrm{m}^{2}$ & $26.42 \pm 3.28$ & $26.59 \pm 3.10$ & 0.552 \\
\hline \multicolumn{4}{|l|}{ Medical measurements } \\
\hline $\mathrm{SBP}, \mathrm{mmHg}$ & $128.08 \pm 16.14$ & $127.70 \pm 14.31$ & 0.793 \\
\hline $\mathrm{DBP}, \mathrm{mmHg}$ & $76.48 \pm 10.71$ & $75.01 \pm 9.60$ & 0.613 \\
\hline \multicolumn{4}{|l|}{ Risk factors } \\
\hline Smoking,n(\%) & $273(51.7)$ & $91(57.2)$ & 0.257 \\
\hline drinkers,n(\%) & $141(26.7)$ & $46(28.9)$ & 0.652 \\
\hline Hypertension,n(\%) & $363(68.8)$ & $107(67.3)$ & 0.804 \\
\hline Dyslipidaemia,n(\%) & $362(68.6)$ & $117(73.6)$ & 0.267 \\
\hline Previous MI,n(\%) & $137(25.9)$ & $56(35.2)$ & 0.029 \\
\hline Past PCI,n(\%) & $181(34.3)$ & $57(35.8)$ & 0.788 \\
\hline Previous Stroke,n(\%) & $47(8.9)$ & $18(11.3)$ & 0.448 \\
\hline $\mathrm{CKD}, \mathrm{n}(\%)$ & $13(2.5)$ & $4(2.5)$ & 0.970 \\
\hline LVEF(\%) & $60 \pm 8.0$ & $59 \pm 10.0$ & 0.100 \\
\hline \multicolumn{4}{|l|}{ Laboratory findings } \\
\hline $\mathrm{TC}(\mathrm{mg} / \mathrm{dL})$ & $147.70 \pm 39.47$ & $150.91 \pm 40.06$ & 0.372 \\
\hline LDL-C (mg/dL) & $85.78 \pm 33.87$ & $87.11 \pm 34.1$ & 0.667 \\
\hline HDL-C (mg/dL) & $37.71 \pm 13.01$ & $37.59 \pm 14.24$ & 0.923 \\
\hline Triglycerides $(\mathrm{mg} / \mathrm{dL})$ & $132.0(91.0,178.0)$ & $147.0(121.0,203.0)$ & $<0.001$ \\
\hline $\mathrm{FPG}(\mathrm{mg} / \mathrm{dL})$ & $111.0(96.0,143.3)$ & $127.0(99.5,166.0)$ & $<0.001$ \\
\hline $\mathrm{GA}, \%$ & $16.0(14.0,19.0)$ & $16.0(14.0,19.0)$ & 0.517 \\
\hline TyG index & $8.96 \pm 0.65$ & $9.25 \pm 0.63$ & $<0.001$ \\
\hline eGFR, mL/min & $94.75 \pm 15.70$ & $93.71 \pm 17.22$ & 0.476 \\
\hline Uric acid,umol/L & $358.65 \pm 88.12$ & $380.19 \pm 99.25$ & 0.009 \\
\hline Medications at discha & & & \\
\hline
\end{tabular}




\begin{tabular}{|c|c|c|c|}
\hline Aspirin, n (\%) & $524(99.2)$ & $158(99.4)$ & 0.867 \\
\hline P2Y12 inhibitors, n (\%) & 347 (65.7) & $113(71.1)$ & 0.246 \\
\hline Statin,n(\%) & $424(80.3)$ & $123(77.4)$ & 0.487 \\
\hline ACEI/ARBs, n (\%) & $175(33.1)$ & 53 (33.3) & 0.965 \\
\hline$\beta$-blockers, n (\%) & $278(52.7)$ & $88(55.3)$ & 0.613 \\
\hline Insulin, n (\%) & $47(8.9)$ & $20(12.6)$ & 0.223 \\
\hline CCB,n(\%) & $64(12.1)$ & $22(13.8)$ & 0.663 \\
\hline \multicolumn{4}{|l|}{ Angiographic findings } \\
\hline One-vessel disease,n(\%) & $98(18.6)$ & $36(22.6)$ & 0.306 \\
\hline Two-vessel disease,n(\%) & $144(27.3)$ & $36(22.6)$ & 0.289 \\
\hline three-vessel disease,n(\%) & $284(53.8)$ & $85(53.5)$ & 0.942 \\
\hline \multicolumn{4}{|l|}{ Target vessel territory } \\
\hline LAD, n (\%) & $198(37.5)$ & $70(44.0)$ & 0.166 \\
\hline LCX, n (\%) & $110(20.8)$ & $29(18.2)$ & 0.548 \\
\hline
\end{tabular}

\begin{tabular}{|llll|}
\hline & Without events $(\mathrm{n}=528)$ & Primary endpoint $(\mathrm{n}=159)$ & P value \\
\hline $\mathrm{RCA}, \mathrm{n}(\%)$ & $302(57.2)$ & $85(53.5)$ & 0.458 \\
\hline
\end{tabular}

\begin{tabular}{|lll|}
\hline & Correlation coefficient & P value \\
\hline Age & -1.06 & 0.006 \\
\hline BMI & 0.268 & $<0.001$ \\
\hline FBG & 0.633 & $<0.001$ \\
\hline GA & 0.260 & $<0.001$ \\
\hline TG & 0.820 & $<0.001$ \\
\hline TC & 0.233 & $<0.001$ \\
\hline LDL-C & 0.097 & 0.011 \\
\hline HDL-C & -0.072 & 0.058 \\
\hline Uric acid & 0.143 & $<0.001$ \\
\hline eGFR & -0.005 & 0.892 \\
\hline LVEF & -0.080 & 0.036 \\
\hline
\end{tabular}




\begin{tabular}{|c|c|c|c|c|}
\hline & $\begin{array}{l}\underline{\text { Univariate analsis }} \\
\underline{\mathrm{HR}}(\underline{95 \% \mathrm{Cl}})\end{array}$ & $P$ value & $\begin{array}{l}\text { Multivariate analsis } \\
\underline{\mathrm{HR}}(\underline{95 \% \mathrm{Cl}}) .\end{array}$ & $P$ value \\
\hline \multicolumn{5}{|l|}{ TyG index tertiles } \\
\hline Q1 & Reference & & Reference & \\
\hline Q2 & $1.675(1.081-2.596)$ & 0.021 & $1.601(1.031-2.486)$ & 0.036 \\
\hline Q3 & $2.827(1.877-4.259)$ & $<0.001$ & $2.560(1.684-3.892)$ & $<0.001$ \\
\hline Age,years & $1.007(0.991-1.023)$ & 0.423 & & \\
\hline Male Sex & $1.181(0.765-1.823)$ & 0.453 & & \\
\hline $\mathrm{BMI}, \mathrm{kg} / \mathrm{m}^{2}$ & $1.022(0.974-1.073)$ & 0.370 & & \\
\hline $\mathrm{SBP}, \mathrm{mmHg}$ & $0.999(0.989-1.009)$ & 0.824 & & \\
\hline $\mathrm{DBP}, \mathrm{mmHg}$ & $0.995(0.981-1.010)$ & 0.532 & & \\
\hline Smoking,n(\%) & $1.167(0.853-1.598)$ & 0.334 & & \\
\hline drinkers,n(\%) & $1.057(0.750-1.490)$ & 0.751 & & \\
\hline Hypertension,n(\%) & $0.945(0.678-1.316)$ & 0.738 & & \\
\hline Dyslipidaemia,n(\%) & 1.403 (0.986-1.998) & 0.060 & & \\
\hline Previous MI,n(\%) & $1.421(1.026-1.967)$ & 0.034 & $1.346(0.968-1.870)$ & 0.077 \\
\hline Past PCI,n(\%) & $1.180(0.853-1.632)$ & 0.318 & & \\
\hline Previous Stroke,n(\%) & $1.283(0.786-2.096)$ & 0.319 & & \\
\hline CKD,n(\%) & $0.890(0.329-2.402)$ & 0.817 & & \\
\hline LVEF(\%) & $0.984(0.967-1.000)$ & 0.050 & & \\
\hline $\mathrm{TC}(\mathrm{mg} / \mathrm{dL})$ & $1.000(0.997-1.004)$ & 0.856 & & \\
\hline LDL-C (mg/dL) & $0.999(0.995-1.004)$ & 0.701 & & \\
\hline $\mathrm{HDL}-\mathrm{C}(\mathrm{mg} / \mathrm{dL})$ & $1.000(0.988-1.011)$ & 0.969 & & \\
\hline $\mathrm{GA}, \%$ & $1.001(0.973-1.029)$ & 0.947 & & \\
\hline eGFR, $\mathrm{mL} / \mathrm{min}$ & $0.995(0.986-1.004)$ & 0.289 & & \\
\hline Uric acid,umol/L & $1.002(1.000-1.003)$ & 0.024 & $1.001(0.999-1.003)$ & 0.230 \\
\hline Aspirin, n (\%) & $1.053(0.147-7.522)$ & 0.959 & & \\
\hline P2Y12 inhibitors, n (\%) & 1.681 (1.189-2.376) & 0.003 & $1.584(1.119-2.244)$ & 0.010 \\
\hline Statin,n(\%) & 1.085 (0.747-1.576) & 0.670 & & \\
\hline
\end{tabular}




\begin{tabular}{|c|c|c|c|c|c|c|}
\hline ACEI/ARBs, $n(\%)$ & \multicolumn{2}{|c|}{$1.104(0.793-1.536)$} & 0.558 & & & \\
\hline ß-blockers, n (\%) & \multicolumn{2}{|c|}{$1.122(0.821-1.534)$} & 0.470 & & & \\
\hline Insulin, n (\%) & \multicolumn{2}{|c|}{$1.789(1.118-2.864)$} & 0.015 & \multicolumn{2}{|c|}{$1.371(0.849-2.216)$} & 0.197 \\
\hline $\mathrm{CCB}, \mathrm{n}(\%)$ & \multicolumn{2}{|c|}{$1.330(0.847-2.088)$} & 0.215 & & & \\
\hline \multicolumn{7}{|l|}{ Angiographic findings } \\
\hline One-vessel disease,n(\%) & \multicolumn{2}{|c|}{$1.229(0.848-1.782)$} & 0.277 & & & \\
\hline Two-vessel disease,n(\%) & \multicolumn{2}{|c|}{$0.787(0.543-1.141)$} & 0.206 & & & \\
\hline three-vessel disease,n(\%) & \multicolumn{2}{|c|}{$1.016(0.744-1.388)$} & 0.919 & & & \\
\hline \multicolumn{7}{|l|}{ Target vessel territory } \\
\hline LAD, n (\%) & \multicolumn{2}{|c|}{$1.229(0.899-1.682)$} & 0.196 & & & \\
\hline LCX, n (\%) & \multicolumn{2}{|c|}{$0.901(0.602-1.347)$} & 0.610 & & & \\
\hline RCA, n (\%) & \multicolumn{2}{|c|}{$0.867(0.635-1.184)$} & 0.370 & & & \\
\hline & \multirow{2}{*}{\multicolumn{2}{|c|}{$\begin{array}{l}\text { Q1 } \\
(n=229)\end{array}$}} & Q2 & \multirow{2}{*}{$\begin{array}{l}\text { Q3 } \\
(n=229)\end{array}$} & \multirow[t]{2}{*}{$P$ value } & \\
\hline & & & $(n=229)$ & & & \\
\hline Primary endpoint, n (\%) & & $33(14.4)$ & $51(22.3)$ & 75 (32.8) & $<0.001$ & \\
\hline Overall death, n (\%) & & $3(1.3)$ & $3(1.3)$ & $4(1.7)$ & 0.904 & \\
\hline Non-fatal stroke, n (\%) & & $4(1.7)$ & $6(2.6)$ & $5(2.2)$ & 0.815 & \\
\hline Non-fatal MI, n (\%) & & $7(3.1)$ & $10(4.4)$ & $13(5.7)$ & 0.390 & \\
\hline Unplanned revascularizati & $n, n(\%)$ & $21(9.2)$ & $31(13.5)$ & $57(24.9)$ & $<0.001$ & \\
\hline
\end{tabular}


Univariable analysis

HR (95\% Cl)
Multivariable analysis ${ }^{c}$

$\mathrm{P} \quad \mathrm{HR}(95 \% \mathrm{Cl})$
$P$ value

TyG index as a nominal variable ${ }^{a}$

Primary endpoint

$\begin{array}{lllll}\text { Q1 } & \text { Reference } & -/- & \text { Reference } & -/- \\ \text { Q2 } & 1.675(1.081- & 0.021 & 1.778(1.124- & 0.014 \\ & 2.596) & & 2.813) & \\ \text { Q3 } & 2.827(1.877- & <0.001 & \begin{array}{l}3.199(2.000- \\ 5.118)\end{array} & <0.001\end{array}$

Overall death

$\begin{array}{lllll}\text { Q1 } & \text { Reference } & -/- & \text { Reference } & \text {-/- } \\ \text { Q2 } & 1.022(0.206- & 0.979 & 0.478(0.032- & 0.590 \\ & 5.062) & & 7.032) & \\ \text { Q3 } & 1.392(0.12-6.221) & 0.665 & 0.293(0.013- & 0.442 \\ & & & 6.727)\end{array}$

Non-fatal stroke

$\begin{array}{lllll}\text { Q1 } & \text { Reference } & -/- & \text { Reference } & -/- \\ \text { Q2 } & 1.735(0.487- & 0.295 & 1.501(0.340- & 0.592 \\ & 6.179) & & 6.627) & \\ \text { Q3 } & 1.363(0.365- & 0.645 & 0.958(0.193- & 0.959 \\ & 5.084) & & 4.752)\end{array}$

Unplanted repeat

revascularization

\begin{tabular}{|lllll|}
\hline Q1 & Reference & $-/-$ & Reference & $-/-$ \\
\hline Q2 & $1.535(0.882-$ & 0.129 & $1.610(0.900-$ & 0.109 \\
& $2.672)$ & & $2.883)$ & \\
\hline Q3 & $\begin{array}{l}3.115(1.888- \\
5.139)\end{array}$ & $<0.001$ & $\begin{array}{l}3.743(2.113- \\
6.630)\end{array}$ & $<0.001$ \\
\hline Q1 & & & \\
\hline Q2 & Reference & $-/-$ & Reference & $-/-$ \\
\hline Q3 & $1.507(0.573-$ & 0.406 & $1.668(0.588-$ & 0.336 \\
& $3.959)$ & & $4.729)$ & \\
\hline & $2.090(0.834-$ & 0.116 & $1.970(0.676-$ & 0.214 \\
& & & $5.741)$ & \\
\hline
\end{tabular}




\begin{tabular}{|lllll|}
$\begin{array}{l}\text { TyG index as a continuous } \\
\text { variable }\end{array}$ & \multicolumn{3}{|c|}{} \\
\hline Primary endpoint & $1.833(1.466-$ & $<0.001$ & $2.168(1.599-$ & $<0.001$ \\
& $2.293)$ & & $2.940)$ & \\
\hline Overall death & $1.698(0.694-$ & 0.246 & $0.783(0.115-$ & 0.803 \\
& $4.152)$ & & $5.349)$ & \\
\hline Non-fatal stroke & $1.153(0.545-$ & 0.710 & $0.920(0.315-$ & 0.879 \\
& $2.437)$ & & $2.688)$ & \\
\hline Unplanned revascularization & $1.919(1.467-$ & $<0.001$ & $2.320(1.609-$ & $<0.001$ \\
& $2.511)$ & & $3.346)$ & \\
\hline Non-fatal Ml & $1.671(0.992-$ & 0.054 & $1.935(0.944-$ & 0.072 \\
& $2.816)$ & & $3.967)$ & \\
\hline
\end{tabular}

TyG triglyceride glucose, MI myocardial infarction, HR hazard ratio, $\mathrm{Cl}$ confidence interval

a The HR was examined regarding Q1 group as reference (stratified by the TyG index tertiles)

b The HR was examined by per 1-unit increase of TyG index

c The multivariate analysis was adjusted for age, male, BMI, SBP, DBP, smoking, drinkers, Hypertension, dyslipidemia, previous MI, past PCl, previous stroke, CKD, LVEF, TC, LDL-C, HDL-C, eGFR, Uric acid, GA, Aspirin, P2Y12 inhibitors, Statin, ACEI/ARBs, $\beta$-blockers, Insulin, CCB, One-vessel disease, Two-vessel disease, three-vessel disease, LAD treatment, LCX treatment, RCA treatment.

\section{Figures}


CTO patients underwent PCI from 2018 January to 2019 December $(\mathrm{n}=1533)$

\section{Patients with diabetes were enrolled:}

(1) Previously diagnosed diabetes under treated with hypoglycemic medication;

(2) Symptoms of diabetes with random blood glucose $\geq 11.1 \mathrm{mmol} / \mathrm{L}$, and $/$ or $F B G \geq 7.0 \mathrm{mmol} / \mathrm{L}$ and/or OGTT $2 \mathrm{~h} \mathrm{PG} \geq 11.1 \mathrm{mmol} / \mathrm{L}$. follow-up after discharge $(\mathrm{n}=856)$

\section{Exclusion criteria:}

(1) Patients with prior CABG, Left ventricular ejection fraction(LVEF) $<30 \%$, renal dysfunction with creatinine clearance $(\mathrm{CrCI})<15 \mathrm{~mL} / \mathrm{min}$ or chronic dialysis;

(2) Extreme body mass index(BMI $\left.>45 \mathrm{~kg} / \mathrm{m}^{2}\right)$, suspected familial hypertriglyceridaemia [plasma triglycerides $\geq 500 \mathrm{mg} / \mathrm{dL}(5.65 \mathrm{mmol} / \mathrm{L})$ ] ;

(3) Missing clinical or follow-up data;

(4) PCI failure, PCI-related complications.

- overall death

- non- fatal stroke

-non-fatal myocardial infarction (MI)

-unplanned repeat revascularization

Primary endpoint $(n=159)$
Final enrolled patients ( $\mathrm{n}=687$ )

TyG index was calculaed

\section{Figure 1}

The flow chart of study population enrollment. CTO chronic total occlusion; $\mathrm{PCI}$ percutaneous coronary intervention; FBG, fasting blood glucose; OGTT, Oral glucose tolerance test; CABG coronary artery bypass grafting, TGs triglycerides; BMI: body mass index, TyG triglyceride glucose. 


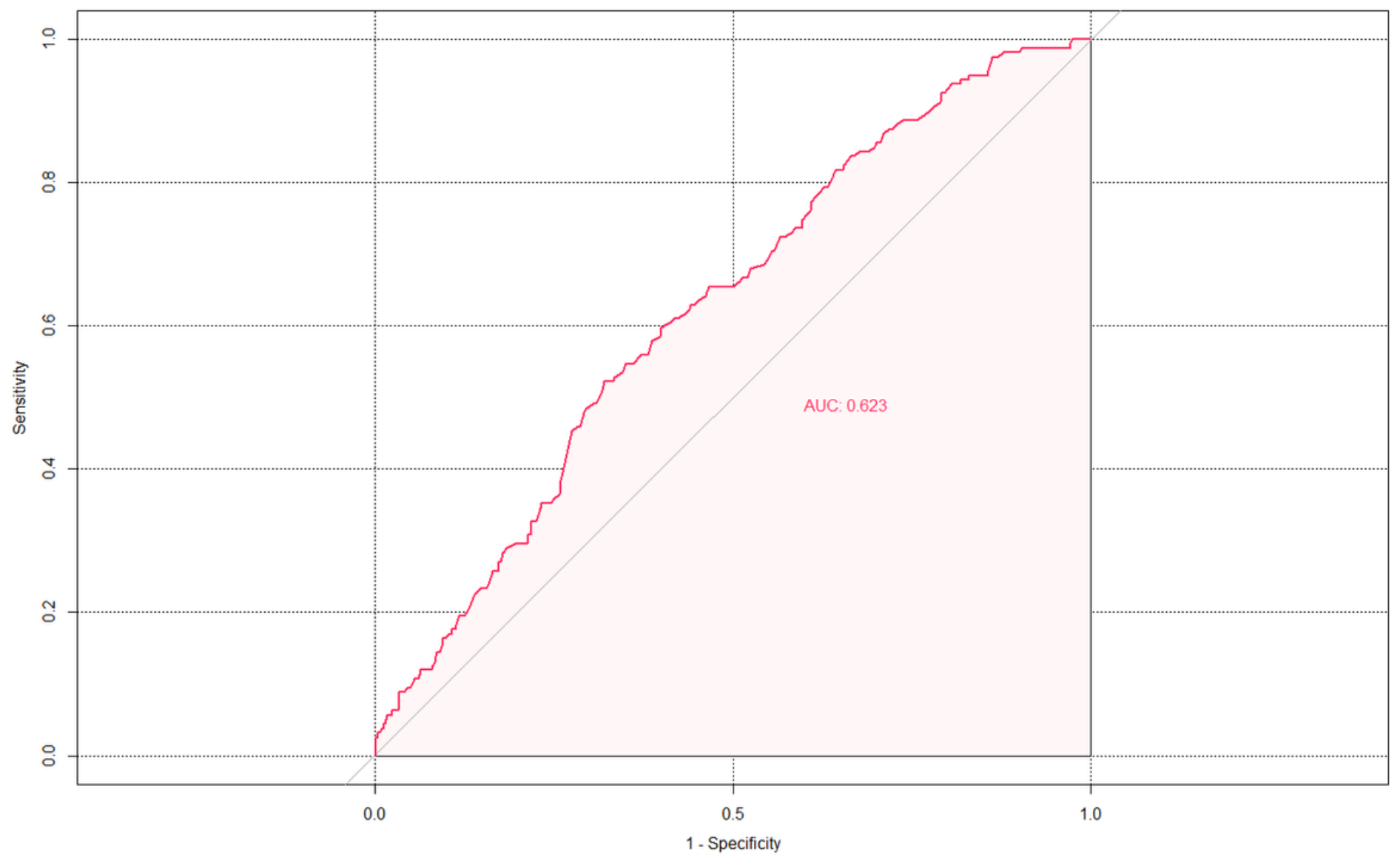

Figure 2

The receiver operating characteristic ( $\mathrm{ROC})$ curves of the triglyceride-glucose index as a marker to predict the primary endpoint in CTO patients after PCl. The area under ROC curves $₫ A U C s \rrbracket o f$ the triglycerideglucose index for predicting the occurrence of the primary endpoint in CTO patients within a median follow-up of 22.0 months after PCl was 0.623 (95\% Cl 0.575-0.671; Pख0.001). 

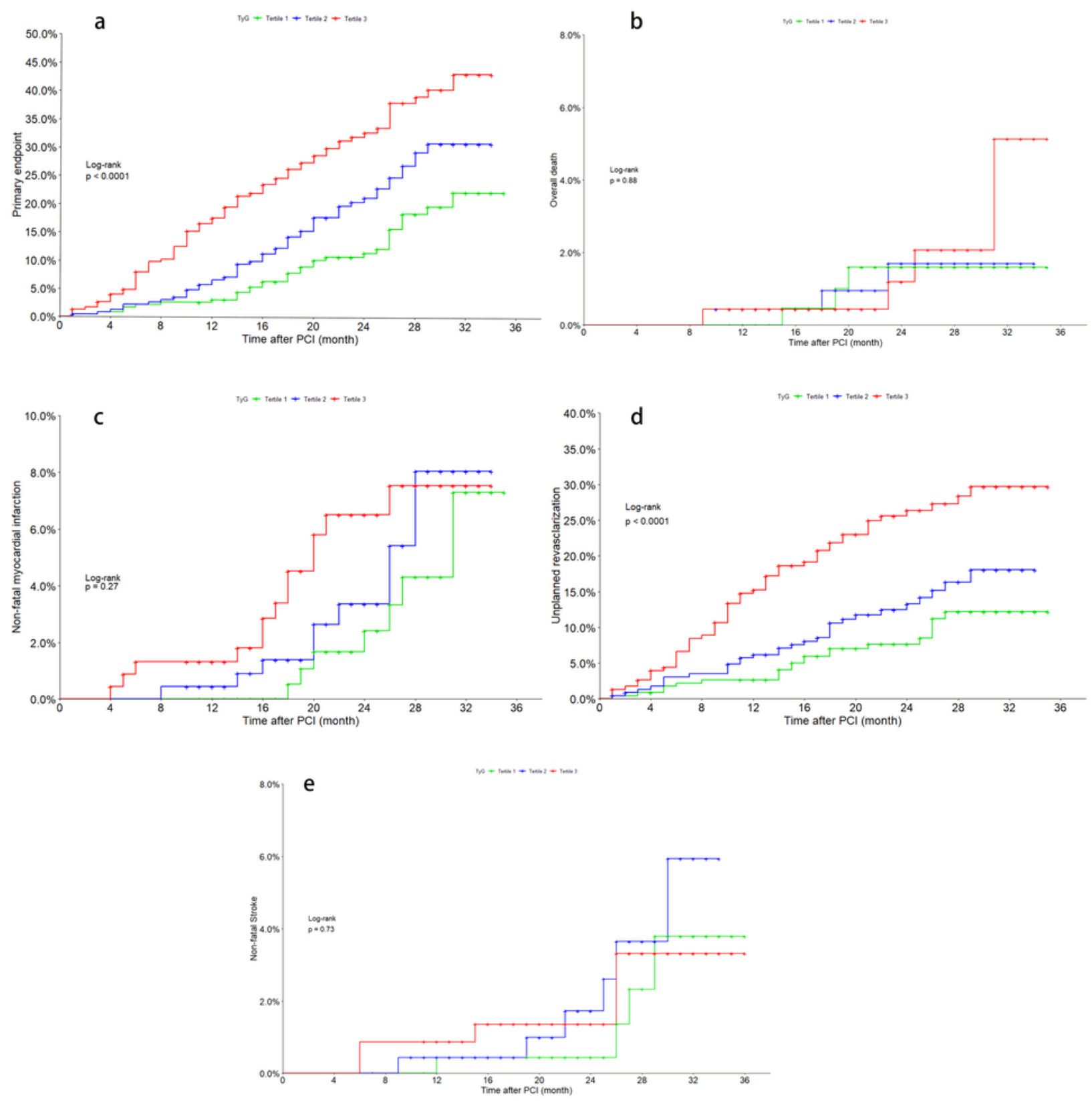

\section{Figure 3}

Kaplan-Meier curves for the primary endpoint (a), overall death (b), non-fatal MI (c), unplanned revascularization (d), non-fatal stroke (e); The groups were stratified by the TyG index tertiles. TyG triglyceride glucose; $\mathrm{Ml}$, myocardial infarction; $\mathrm{HR}$, hazard ratio; $\mathrm{Cl}$, confidence interval. 